\title{
Mechanical Equations on Slant Curves in 3-dimensional Normal Almost Paracontact Metric Manifolds
}

\author{
Oğuzhan Çelik ${ }^{1}$, Zeki Kasap ${ }^{2}$ \\ ${ }^{I}$ Department of Mathematics, University of Çanakkale Onsekizmart, Çanakkale, TURKEY \\ ${ }^{2}$ Department of Elementary, Mathematics Education, Pamukkale University, Denizli, TURKEY
}

*Corresponding Author: Zeki Kasap, Department of Elementary, Mathematics Education, Pamukkale University, Denizli, TURKEY

\begin{abstract}
It is well known that an almost paracontact manifold is an analogue of the almost contact manifold and it is closely related to almost complex manifold. An almost contact manifold is always odd dimensional on the other hand an almost paracontact manifold could be even dimensional as well. Slant curves are characterized through the scalar product between the normal at the curve and the vertical vector field. The classic mechanic was built on vectors. A formulation of classical mechanics is Lagrangian and Hamiltonian mechanics. The purpose of this paper is the to study Lagrangian and Hamiltonian formalism for mechanical systems using slant curves in 3-dimensional normal almost paracontact metric manifolds, which speaks for a quirky multidisciplinary fields of research. Furthermore, in this study geometricals and physical results concerning to on slant curves in 3-dimensional normal almost paracontact metric manifolds are given.
\end{abstract}

Keywords: Symplectic Geometry, Lagrangian, Hamiltonian, Mechanical System.

\section{INTRODUCTION}

There are many studies about Lagrangian and Hamiltonian dynamics, mechanics, formalisms, systems and equations. There are real, complex, para-complex and other analogues. It is well-known that Lagrangian and Hamiltonian analogues are very important tools. They have a simple method to describe the model for mechanical systems. Welyczko generalized some of them to the case of 3dimensional normal almost contact metric manifolds, especially, quasi-Sasakian manifolds [1]. Tripathi et al. introduce the concept of $\varepsilon$-almost paracontact manifolds, and in particular, of $\varepsilon$-paraSasakian manifolds [2]. Atceken investigated the existence of warped product semi-invariant submanifolds in almost para contact metric manifolds [3]. Calin et al. studied slant curves of threedimensional f-Kenmotsu manifolds [4]. Calin and Crasmareanu examined slant curves of threedimensional normal almost contact manifolds as natural generalization of Legendre curves [5]. Kasap and Tekkoyun found Lagrangian and Hamiltonian formalism for mechanical systems using para/pseudo-Kähler manifolds [6]. Guvenc and Ozgur find the characterizations of the curvatures of slant curves in trans-Sasakian manifolds with C-parallel [7]. Welyczko studied the curvature and torsion of slant Frenet curves in 3-dimensional normal almost paracontact metric manifolds [8].

In the present paper, we present equations related to Lagrangian and Hamiltonian mechanical systems on Slant curves in 3-dimensional normal almost paracontact metric manifolds.

\section{PReliminaries}

Definition 1. A $(2 n+1)$-dimensional manifold $M$ is said to be a contact manifold if it admits a global 1 -form $\eta$, such that $\eta \wedge(d \eta)^{\mathrm{n}} \neq 0$.

Given such a form $\eta$, there exists a unique vector field $\xi$, called the characteristic vector field, such that $\eta(\xi)=1$ and $\operatorname{d} \eta(\xi)=$,0 . A semi-Riemannian metric $\mathrm{g}$ is said to be an associated metric if there exists a tensor $\phi$ of type $(1,1)$, such that

$$
\phi^{2} \mathrm{X}=\mathrm{X}-\eta(\mathrm{X}) \xi
$$




$$
\begin{aligned}
& \phi \xi=0, \eta(\phi X)=0, \eta(\xi)=1, \\
& g(\phi X, \phi Y)=g(X, Y)-\eta(X) \eta(Y), \\
& \eta(X)=g(X, \xi), d \eta(,)=g(, \phi) .
\end{aligned}
$$

Then, $(\phi, \xi, \eta, \mathrm{g})$ (more briefly, $(\eta, \mathrm{g}))$ is called a paracontact metric structure, and $(\mathrm{M}, \phi, \xi, \eta, \mathrm{g})$ or $\mathrm{M}$ a paracontact metric manifold [9].

Definition 2. Let $M$ be an almost paracontact manifold with almost paracontact structure $(\phi, \xi, \eta, g)$ and consider the product manifold $M \times \mathbb{R}$, where $\mathbb{R}$ is the real line. A vector field on $M \times \mathbb{R}$ can be represented by $(X, f(d / d t))$, where $X$ is tangent to $M, f$ a smooth function on $M \times \mathbb{R}$, and the coordinates of $\mathbb{R}$. For any two vector fields $(\mathrm{X}, \mathrm{f}(\mathrm{d} / \mathrm{dt}))$ and $(\mathrm{Y}, \mathrm{h}(\mathrm{d} / \mathrm{dt}))$, it is easy to verify the following:

$$
[(X, f(d /(d t))),(Y, h(d /(d t))]=([X, Y],(X h-Y f)(d /(d t))) .
$$

Definition 3. If the induced almost product structure $\mathrm{J}$ on $\mathrm{M} \times \mathbb{R}$ defined by

$$
J(X, f(d /(d t)))=(\phi X+f \xi, \eta(X)(d /(d t)))
$$

is integrable, then we say that the almost paracontact structure $(\phi, \xi, \eta, g)$ is normal.

Definition 4. Let $M$ be an almost paracontact manifold and for any vector fields $X, Y$ on $M$ if it is additionaly endowed with a pseudo-Riemann metric $g$ of signature $(n+1, n)$ and such that

$$
\mathrm{g}(\phi X, \phi \mathrm{Y})=-\mathrm{g}(\mathrm{X}, \mathrm{Y})+\eta(\mathrm{X}) \eta(\mathrm{Y}) .
$$

Definition 5. In normal almost contact metric manifold with Riemanian metric $g$, the value of $g(\gamma, \xi)$ satisfied $-1 \leq \mathrm{g}(\gamma, \xi) \leq 1$, so that we can define the structural angel of $\gamma$ i.e. function $\theta: \mathrm{I} \rightarrow[0,2 \pi)$ given by

$$
\cos \theta(\mathrm{t})=\mathrm{g}(\dot{\gamma}(\mathrm{t}), \xi)=\eta(\dot{\gamma}(\mathrm{t})) .
$$

Then, the curve $\gamma$ is said to be a slant curve, (or $\theta$ slant curve) if $\theta$ is a constant function.

\section{THE THEORY OF J-HOLOMORPHIC CURVES}

The theory of J-holomorphic curves is one of the new techniques which have recently revolutionized the study of symplectic geometry, making it possible to study the global structure of symplectic manifolds. The methods are also of interest in the study of Kähler manifolds, since often when one studies properties of holomorphic curves in such manifolds it is necessary to perturb the complex structure to be generic. The effect of this is to ensure that one is looking at persistent rather than accidental features of these curves.

Symplectic manifolds arise naturally in abstract formulations of classical mechanics and analytical mechanics as the cotangent bundles of manifolds, e.g., in the Hamiltonian formulation of classical mechanics, which provides one of the major motivations for the field: The set of all possible configurations of a system is modelled as a manifold, and this manifold's cotangent bundle describes the phase space of the system.

Definition 6. J-holomorphic curve is a smooth map from a Riemann surface into an almost complex manifold that satisfies the Cauchy-Riemann equation.

Definition 7. A symplectic manifold is a smooth manifold (M) equipped with a closed nondegenerate differential 2-form $(\omega)$ called the symplectic form.

Example 1. An almost complex symplectic manifold is standard Euclidean space $\left(\mathbb{R}^{2 \mathrm{n}}, \omega_{0}\right)$ with its standard almost complex structure $\mathrm{J}_{0}$ obtained from the usual identification with $\mathbb{C}_{\mathrm{n}}$. Thus, one sets $z_{j}=x_{2 j-1}+i x_{2 j}$ for $j=1, \ldots, n$ and defines $J_{0}$ by

$$
\mathrm{J}_{0}\left(\partial_{2 \mathrm{j}-1}\right)=\partial_{2 \mathrm{j}}, \quad \mathrm{J}_{0}\left(\partial_{2 \mathrm{j}}\right)=-\partial_{2 \mathrm{j}-1}
$$


where $\partial_{\mathrm{j}}=\partial / \partial \mathrm{x}_{\mathrm{j}}$ is the standard basis of $\mathrm{T}_{\mathrm{x}} \mathbb{R}^{2 \mathrm{n}}[10]$.

Definition 8. Let $M$ be a differentiable manifold of dimension $(2 n+1)$ and suppose $J$ is a differentiable vector bundle isomorphism $\mathrm{J}: \mathrm{TM} \rightarrow \mathrm{TM}$ such that $\mathrm{J}: \mathrm{TM} \rightarrow \mathrm{TM}$ is a almost complex structure for $\mathrm{TM}$.

Definition 9. An almost complex structure $J$ on $M$ assigns to each $p \in M$ a linear map $J_{p}: T_{p} M \rightarrow T_{p} M$ that is smooth in $\mathrm{p}$ and satisfies $\mathrm{J}_{\mathrm{p}}^{2}=\mathrm{Id}$ for all $\mathrm{p}$. The pair $(\mathrm{M}, \mathrm{J})$ is called an almost paracomplex manifold.

Theorem 1. Any paracomplex manifold $\mathrm{M}$ is also an almost paracomplex manifold.

Lemma 1. Let $M$ be a smooth manifold. If $M$ admits a complex structure $A$, then $M$ admits an almost complex structure $\mathrm{J}$. Let $\operatorname{dim}_{\mathbb{C}} \mathrm{M}=\mathrm{m}$ and $(\mathrm{z}, \mathrm{U})$ be any holomorphic chart inducing a coordinate frame $\partial \mathrm{x}_{1}, \partial \mathrm{y}_{1}, \ldots, \partial \mathrm{x}_{\mathrm{m}}, \partial \mathrm{y}_{\mathrm{m}}$. Then $\mathrm{J}$ is given locally as

$$
\mathrm{J}_{\mathrm{p}}\left(\partial \mathrm{x}_{\mathrm{i}} \mid \mathrm{p}\right)=\partial \mathrm{y}_{\mathrm{i}}\left|\mathrm{p}, \quad \mathrm{J}_{\mathrm{p}}\left(\partial \mathrm{y}_{\mathrm{i}} \mid \mathrm{p}\right)=-\partial \mathrm{x}_{\mathrm{i}}\right| \mathrm{p},
$$

where $1 \leq \mathrm{i} \leq \mathrm{m}$ and $\mathrm{p} \in \mathrm{U}[11]$.

A celebrated theorem of Newlander and Nirenberg [12] says that an almost (para)complex structure is a (para)complex structure if and only if its Nijenhuis tensor or torsion vanishes.

Theorem 2. The almost (para)complex structure $J$ on $M$ is integrable if and only if the tensor $N_{J}$ vanishes identically, where $\mathrm{N}_{\mathrm{J}}$ is defined on two vector fields $\mathrm{X}$ and $\mathrm{Y}$ by

$$
\mathrm{N}_{\mathrm{J}}[\mathrm{X}, \mathrm{Y}]=[\mathrm{JX}, \mathrm{JY}]-\mathrm{J}[\mathrm{X}, \mathrm{JY}]-\mathrm{J}[\mathrm{JX}, \mathrm{Y}]-[\mathrm{X}, \mathrm{Y}] \text {. }
$$

The tensor $(2,1)$ is called the Nijenhuis tensor (8). We say that $J$ is torsion free if $N_{J}=0$. An almost product structure is integrable if its Nijenhuis tensor vanishes.

Theorem 3. (Newlander-Nirenberg) An almost complex manifold (M,J) is complex if and only if $\mathbf{J}$ is integrable.

Slant curves are characterized through the scalar product between the normal at the curve and the vertical vector field $[4,5]$.

Definition 10. In three dimensions, the vector from the origin to the point with cartesian coordinates $(\mathrm{x}, \mathrm{y}, \mathrm{z})$ can be written as [13]: $\mathrm{r}=\mathrm{x} \cdot \vec{i}+\mathrm{y} \cdot \vec{j}+\mathrm{z} \cdot \vec{k}=\mathrm{x}(\partial /(\partial \mathrm{x}))+\mathrm{y}(\partial /(\partial \mathrm{y}))+\mathrm{z}(\partial /(\partial \mathrm{z}))$.

Example 2. Let $\mathbb{R}^{3}$ be the Cartesian space and $(x, y, z)$ be the cartesian coordinates in it. define the standart almost paracontact structure $(\phi, \xi, \eta)$ on $\mathbb{R}^{3}$ by

$$
\begin{aligned}
& \phi\left(\partial_{1}\right)=\partial_{2}-2 x \partial_{3}, \\
& \phi\left(\partial_{2}\right)=\partial_{1}, \phi\left(\partial_{3}\right)=0 \\
& \xi=\partial_{3}, \eta=2 x d y+d z,
\end{aligned}
$$

where $\phi(\partial /(\partial \mathrm{x}))=(\partial /(\partial \mathrm{y}))-2 \mathrm{x}(\partial /(\partial \mathrm{z})), \phi(\partial /(\partial \mathrm{y}))=\partial /(\partial \mathrm{x}), \phi(\partial /(\partial \mathrm{z}))=0$ for $\partial_{1}=\partial /(\partial \mathrm{x}), \partial_{2}=\partial /(\partial \mathrm{y}), \partial_{3}=\partial /(\partial \mathrm{z})$

$$
\begin{aligned}
& \text { 1. } \phi^{2}(\partial /(\partial \mathrm{x}))=\phi\left((\partial /(\partial \mathrm{y}))-2 \mathrm{x} \phi(\partial /(\partial \mathrm{z}))=\partial /(\partial \mathrm{x}), \quad \phi^{2}=\mathrm{I},\right. \\
& \text { 2. } \phi^{2}(\partial /(\partial \mathrm{y}))=(\partial /(\partial \mathrm{y}))-2 \mathrm{x}(\partial /(\partial \mathrm{z})), \phi^{2}=\mathrm{I}-\eta(\mathrm{X}) \xi .
\end{aligned}
$$

\section{EUler-LAgrange DyNAMics EQUATIONS}

Definition 11. Let $M$ be an $n$-dimensional manifold and TM its tangent bundle with canonical projection $\tau_{\mathrm{M}}: \mathrm{TM} \rightarrow \mathrm{M}$. TM is called the phase space of velocities of the base manifold $\mathrm{M}$. Let $\mathrm{L}: \mathrm{TM} \rightarrow \mathbb{R}$ be a differentiable function on TM called the Lagrangian function $[14,15]$. 
Here, $\mathrm{L}=\mathrm{T}-\mathrm{V}$ such that $\mathrm{T}$ is the kinetic energy and $\mathrm{V}$ is the potential energy of a mechanical system. In the problem of a mass on the end of a spring, $\mathrm{T}=\mathrm{m} \dot{x}^{2} / 2$ and $\mathrm{V}=\mathrm{kx} x^{2} / 2$, so we have $\mathrm{L}=\left(\mathrm{m} \dot{x}^{2}\right) / 2-\left(\mathrm{kx}^{2}\right) / 2$. We consider the closed 2-form and base space $(J)$ on TM given by $\Phi_{\mathrm{L}}=-\mathrm{d}\left(\mathbf{d}_{\mathrm{J}} \mathrm{L}\right)=-\mathrm{d}(\mathrm{J}(\mathbf{d}))$. Consider the equation

$$
\mathrm{i}_{\xi} \Phi_{\mathrm{L}}=\mathrm{dE}_{\mathrm{L}}
$$

Where $i_{\xi}$ is reduction function and $i_{\xi} \Phi_{L}=\Phi_{L}(\xi)$ is defined in the form. Then $\xi$ is a vector field, we shall see that (11) under a certain condition on $\xi$ is the intrinsical expression of the Euler-Lagrange equations of motion. This equation (11) is named as Lagrange dynamical equation.

Definition 12. We shall see that for motion in a potential, $E_{L}=V L-L$ is an energy function and $V=J \xi$ a Liouville vector field. Here $\mathrm{dE}_{\mathrm{L}}$ denotes the differential of $\mathrm{E}$. The triple $\left(\mathrm{TM}, \Phi_{\mathrm{L}}, \xi\right)$ is known as Lagrangian system on the tangent bundle TM. If it is continued the operations on (11) for any coordinate system then infinite dimension Lagrange's equation is obtained the form below. The equations of motion in Lagrangian mechanics are the Lagrange equations of the second kind, also known as the Euler-Lagrange equations;

$$
(\partial /(\partial \mathrm{t}))((\partial \mathrm{L}) /(\partial \dot{x}))=(\partial \mathrm{L}) /(\partial \mathrm{x}) .
$$

Definition 13. We have $(\partial \mathrm{L}) /(\partial \dot{x})=\mathrm{mx}$ and $(\partial \mathrm{L}) /(\partial \mathrm{x})=-\mathrm{kx}$, so eq. (12) gives $m \ddot{x}=-\mathrm{kx}$ which is exactly the result obtained by using $\mathrm{F}=\mathrm{ma}$ at Newton's second law for the mechanical problem. The EulerLagrange equation, eq. (12), gives $m \ddot{x}=-(\mathrm{dV}) /(\mathrm{dx})$. In a three-dimensional setup written in terms of cartesian coordinates, the potential takes the form $\mathrm{V}(\mathrm{x}, \mathrm{y}, \mathrm{z})$, so the Lagrangian is $\mathrm{L}=\mathrm{m}\left(\dot{x}^{2}+\dot{y}^{2}+\dot{z}^{2}\right.$ )/2-V(x,y,z). So, the three Euler-Lagrange equations may be combined into the vector statement $m \ddot{x}$ $=-\nabla \mathrm{V}$.

\section{HAMILTON DYNAMiCS EQUATIONS}

The Lagrangian formulation is an important springboard from which to develop another useful formulation of classical mechanics known as the Hamiltonian formulation. The Hamiltonian of a system is defined to be the sum of the kinetic and potential energies expressed as a function of positions and their conjugate momenta.

Definitions 14. Let $M$ is the configuration manifold and its cotangent manifold $T^{*} M$. By a symplectic form we mean a 2-form $\Phi$ on $\mathrm{T} * \mathrm{M}$ such that:

(i) $\Phi$ is closed, that is, $d \Phi=0$; (ii) for each $z \in T * M, \Phi: T * M \times T * M \rightarrow \mathbb{R}$ is weakly nondegenerate. If $\Phi_{z}$ in (ii) is nondegenerate, we speak of a strong symplectic form. If (ii) is dropped we refer to $\Phi$ as a presymplectic form. Let $(T * M, \Phi)$ be a symplectic manifold. A vector field $Z_{H}$ : $T * M \rightarrow T * M$ is called Hamiltonian if there is a $\mathrm{C}^{1}$ function $\mathrm{H}: \mathrm{T}^{*} \mathrm{M} \rightarrow \mathbb{R}$ such that dynamical equation is determined by

$$
i_{Z_{H}} \Phi=\mathrm{dH}
$$

We say that $Z_{\mathrm{H}}$ is locally Hamiltonian vector field if $\mathrm{i}_{\mathrm{ZH}_{\mathrm{H}}} \Phi$ is closed and where $\Phi$ shows the canonical symplectic form so that $\Phi=-\mathrm{d} \Omega, \Omega=\mathrm{J} *(\omega), \mathrm{J} *$ a dual of $\mathrm{J}, \omega$ a 1 -form on $\mathrm{T} * \mathrm{M}$. The trio $\left(\mathrm{T} * \mathrm{M}, \Phi, \mathrm{Z}_{\mathrm{H}}\right)$ is named Hamiltonian system which it is defined on the cotangent bundle $\mathrm{T} * \mathrm{M}[14,15]$.

Recall from elementary physics that momentum of a particle, $\mathrm{p}_{\mathrm{i}}$, is defined in terms of its velocity $\mathrm{q}_{\mathrm{i}}$ by $\mathrm{p}_{\mathrm{i}}=m_{i} \dot{q}_{i}$. In fact, the more general definition of conjugate momentum, valid for any set of coordinates, is given in terms of the Lagrangian: $\mathrm{p}_{\mathrm{i}}=(\partial \mathrm{L}) /\left(\partial \dot{q}_{i}\right), \dot{p}_{i}=\left((\partial \mathrm{L}) /\left(\partial \mathrm{q}_{\mathrm{i}}\right)\right)$. Note that these two definitions are equivalent for Cartesian variables. In terms of Cartesian momenta, the kinetic energy is 
given by $\mathrm{T}=\sum_{i=1}^{n} p_{i}^{2} / 2 m_{i}$. Then, the Hamiltonian, which is defined to be the sum, $\mathrm{H}=\mathrm{T}+\mathrm{V}$, expressed as a function of positions and momenta, will be given by $\mathrm{H}\left(\mathrm{q}_{\mathrm{i}}, \mathrm{p}_{\mathrm{i}}\right)=\sum_{i=1}^{n} p_{i}^{2} / 2 m_{i}$ $\left.+\mathrm{V}\left(\mathrm{q}_{1}, \ldots, \mathrm{q}_{\mathrm{n}}\right\}\right)$ where $\mathrm{p}=\mathrm{p}_{1}, \ldots, \mathrm{p}_{\mathrm{n}}$. The function $\mathrm{H}$ is equal to the total energy of the system. In terms of the Hamiltonian, the equations of motion of a system are given by Hamilton's equations:

$$
\dot{q}_{i}=(\partial \mathrm{H}) /\left(\partial \mathrm{p}_{\mathrm{i}}\right), \dot{p}_{i}=-(\partial \mathrm{H}) /\left(\partial \mathrm{q}_{\mathrm{i}}\right) .
$$

The solution of Hamilton's equations of motion will yield a trajectory in terms of positions and momenta as functions of time. Hamilton's equations can be easily shown to be equivalent to Newton's equations, and, like the Lagrangian formulation, Hamilton's equations can be used to determine the equations of motion of a system in any set of coordinates.

\section{Euler-Lagrange Mechanical Systems}

Lemma 2. The closed 2-form on a vector field and 1-form reduction function on the phase space defined of a mechanical system is equal to the differential of the energy function 1-form of the Lagrangian and the Hamiltonian mechanical systems [16, 17].

Theorem 2. If $\alpha$ and $\beta$ are 1 -forms, then $\alpha \wedge \beta$ is a 2-forms.

Definitions 15. The vector field $X$ on $T^{*} M$ given by $i_{X} \omega=d H$ is called the geodesic flow of the metric g.

Definitions 16. If $\gamma:(a, b) \rightarrow T * M$ is an integral curve of the geodesic flow, then the curve $p(\gamma)$ in $M$ is called a geodesic.

We, using Lemma 2, get Euler-Lagrange equations for quantum and classical mechanics on 3dimensional normal almost paracontact metric manifolds.

Let take $\phi$ be as the local basis element on 3-dimensional normal almost-paracontact metric manifolds and $(\mathrm{x}, \mathrm{y}, \mathrm{z})$ be its coordinate functions on 3-dimensional normal almost-paracontact metric manifolds;

$$
\phi(\partial /(\partial \mathrm{x}))=\partial /(\partial \mathrm{y})-2 \mathrm{x}(\partial /(\partial \mathrm{z})), \phi(\partial /(\partial \mathrm{y}))=\partial /(\partial \mathrm{x}), \phi(\partial /(\partial \mathrm{z}))=0 .
$$

Proposition 1. Let $\xi$ be the vector field decided by

$$
\xi=\mathrm{X}(\partial /(\partial \mathrm{x}))+\mathrm{Y}(\partial /(\partial \mathrm{y}))+\mathrm{Z}(\partial /(\partial \mathrm{z}))
$$

Then the following differential equations are obtained.

$$
\begin{aligned}
& \text { dif1. }(\partial /(\partial \mathrm{t}))((\partial \mathrm{L}) /(\partial \mathrm{y}))+(\partial /(\partial \mathrm{t}))(2 \mathrm{x}((\partial \mathrm{L}) /(\partial \mathrm{z})))+(\partial \mathrm{L}) /(\partial \mathrm{x})=0, \\
& \text { dif2. }-(\partial /(\partial \mathrm{t}))(((\partial \mathrm{L}) /(\partial \mathrm{x})))+((\partial \mathrm{L}) /(\partial \mathrm{y}))=0, \\
& \text { dif3. }((\partial \mathrm{L}) /(\partial \mathrm{z})) \mathrm{dz}=0,
\end{aligned}
$$

Proof: The vector field described by

$$
\mathrm{V}=\phi(\xi)=\mathrm{X}(\partial /(\partial \mathrm{y}))-2 \mathrm{xX}(\partial /(\partial \mathrm{z}))+\mathrm{Y}(\partial /(\partial \mathrm{x}))
$$

is said to be Liouville vector field on 3-dimensional normal almost-paracontact metric manifolds. The 3-dimensional normal almost-paracontact metric manifolds form is the closed 2-form which is given by $\Phi_{\mathrm{L}}=-\mathrm{d}\left(\mathbf{d}_{\phi} \mathrm{L}\right)$ such that

$$
\mathbf{d}_{\phi}=((\partial /(\partial \mathrm{y}))-2 \mathrm{x}(\partial /(\partial \mathrm{z}))) \mathrm{dx}+(\partial /(\partial \mathrm{x})) \mathrm{dy}
$$


Then we have

$$
\begin{aligned}
& \Phi_{\mathrm{L}}=-\mathrm{d}\left(\mathbf{d}_{\phi} \mathrm{L}\right)=-\mathrm{d}(((\partial \mathrm{L}) /(\partial \mathrm{y})-2 \mathrm{x}((\partial \mathrm{L}) /(\partial \mathrm{z})) \mathrm{d} \mathrm{x}+((\partial \mathrm{L}) /(\partial \mathrm{x})) \mathrm{dy}) \\
&=--((\partial /(\partial \mathrm{x})) \mathrm{d} \mathrm{x}+(\partial /(\partial \mathrm{y})) \mathrm{dy}+(\partial /(\partial \mathrm{z})) \mathrm{dz})(((\partial /(\partial \mathrm{y}))-2 \mathrm{x}(\partial /(\partial \mathrm{z}))) \mathrm{d} \mathrm{x}+(\partial /(\partial \mathrm{x})) \mathrm{dy}) \\
&-\left(\left(\partial^{2} \mathrm{~L}\right) /(\partial \mathrm{x} \partial \mathrm{y})\right) \mathrm{d} \mathrm{x} \wedge \mathrm{d} \mathrm{x}+2 \mathrm{x}\left(\left(\partial^{2} \mathrm{~L}\right) /(\partial \mathrm{x} \partial \mathrm{z})\right) \mathrm{dx} \wedge \mathrm{dx}-\left(\left(\partial^{2} \mathrm{~L}\right) /(\partial \mathrm{x} \partial \mathrm{x})\right) \mathrm{dx} \wedge \mathrm{dy} \\
&-\left(\left(\partial^{2} \mathrm{~L}\right) /(\partial \mathrm{y} \partial \mathrm{y})\right) \mathrm{dy} \wedge \mathrm{dx}+2 \mathrm{x}\left(\left(\partial^{2} \mathrm{~L}\right) /(\partial \mathrm{y} \partial \mathrm{z})\right) \mathrm{dy} \wedge \mathrm{dx}-\left(\left(\partial^{2} \mathrm{~L}\right) /(\partial \mathrm{y} \partial \mathrm{x})\right) \mathrm{dy} \wedge \mathrm{dy} \\
&-\left(\left(\partial^{2} \mathrm{~L}\right) /(\partial \mathrm{z} \partial \mathrm{y})\right) \mathrm{dz} \wedge \mathrm{dx}+2 \mathrm{x}\left(\left(\partial^{2} \mathrm{~L}\right) /(\partial \mathrm{z} \partial \mathrm{z})\right) \mathrm{dz} \wedge \mathrm{dx}-\left(\left(\partial^{2} \mathrm{~L}\right) /(\partial \mathrm{z} \partial \mathrm{x})\right) \mathrm{dz} \wedge \mathrm{dy} .
\end{aligned}
$$

We calculate

$$
\begin{aligned}
\mathrm{i}_{\xi} \Phi_{\mathrm{L}}=\Phi_{\mathrm{L}}(\xi) \\
=\left(-\left(\left(\partial^{2} \mathrm{~L}\right) /(\partial \mathrm{x} \partial \mathrm{y})\right) \mathrm{dx} \wedge \mathrm{dx}+2 \mathrm{x}\left(\left(\partial^{2} \mathrm{~L}\right) /(\partial \mathrm{x} \partial \mathrm{z})\right) \mathrm{dx} \wedge \mathrm{dx}-\left(\left(\partial^{2} \mathrm{~L}\right) /(\partial \mathrm{x} \partial \mathrm{x})\right) \mathrm{dx} \wedge d \mathrm{dy}-\left(\left(\partial^{2} \mathrm{~L}\right) /(\partial \mathrm{y} \partial \mathrm{y})\right) \mathrm{dy} \wedge \mathrm{dx}\right. \\
+2 \mathrm{x}\left(\left(\partial^{2} \mathrm{~L}\right) /(\partial \mathrm{y} \partial \mathrm{z})\right) \mathrm{dy} \wedge \mathrm{dx}-\left(\left(\partial^{2} \mathrm{~L}\right) /(\partial \mathrm{y} \partial \mathrm{x})\right) \mathrm{dy} \wedge \mathrm{dy}-\left(\left(\partial^{2} \mathrm{~L}\right) /(\partial \mathrm{z} \partial \mathrm{y})\right) \mathrm{dz} \wedge \mathrm{dx}+2 \mathrm{x}\left(\left(\partial^{2} \mathrm{~L}\right) /(\partial \mathrm{z} \partial \mathrm{z})\right) \mathrm{dz} \wedge \mathrm{dx} \\
\left.-\left(\left(\partial^{2} \mathrm{~L}\right) /(\partial \mathrm{z} \partial \mathrm{x})\right) \mathrm{dz} \wedge \mathrm{dy}\right)(\mathrm{X}(\partial /(\partial \mathrm{x}))+\mathrm{Y}(\partial /(\partial \mathrm{y}))+\mathrm{Z}(\partial /(\partial \mathrm{z}))) \\
=-\left(\left(\partial^{2} \mathrm{~L}\right) /(\partial \mathrm{x} \partial \mathrm{y})\right) \mathrm{Xdx}+\left(\left(\partial^{2} \mathrm{~L}\right) /(\partial \mathrm{x} \partial \mathrm{y})\right) \mathrm{Xdx}+2 \mathrm{x}\left(\left(\partial^{2} \mathrm{~L}\right) /(\partial \mathrm{x} \partial \mathrm{z})\right) \mathrm{Xdx}-2 \mathrm{x}\left(\left(\partial^{2} \mathrm{~L}\right) /(\partial \mathrm{x} \partial \mathrm{z})\right) \mathrm{Xdx} \\
-\left(\left(\partial^{2} \mathrm{~L}\right) /(\partial \mathrm{x} \partial \mathrm{x})\right) \mathrm{Xdy}+\left(\left(\partial^{2} \mathrm{~L}\right) /(\partial \mathrm{y} \partial \mathrm{y})\right) \mathrm{Xdy}-2 \mathrm{x}\left(\left(\partial^{2} \mathrm{~L}\right) /(\partial \mathrm{y} \partial \mathrm{z})\right) \mathrm{Xdy}+\left(\left(\partial^{2} \mathrm{~L}\right) /(\partial \mathrm{z} \partial \mathrm{y})\right) \mathrm{Xdz} \\
-2 \mathrm{x}\left(\left(\partial^{2} \mathrm{~L}\right) /(\partial \mathrm{z} \partial \mathrm{z})\right) \mathrm{Xdz}+\left(\left(\partial^{2} \mathrm{~L}\right) /(\partial \mathrm{x} \partial \mathrm{x})\right) \mathrm{Ydx}-\left(\left(\partial^{2} \mathrm{~L}\right) /(\partial \mathrm{y} \partial \mathrm{y})\right) \mathrm{Ydx}+2 \mathrm{x}\left(\left(\partial^{2} \mathrm{~L}\right) /(\partial \mathrm{y} \partial \mathrm{z})\right) \mathrm{Ydx} \\
-\left(\left(\partial^{2} \mathrm{~L}\right) /(\partial \mathrm{y} \partial \mathrm{x})\right) \mathrm{Ydy}+\left(\left(\partial^{2} \mathrm{~L}\right) /(\partial \mathrm{y} \partial \mathrm{x})\right) \mathrm{Ydy}+\left(\left(\partial^{2} \mathrm{~L}\right) /(\partial \mathrm{z} \partial \mathrm{x})\right) \mathrm{Ydz}-\left(\left(\partial^{2} \mathrm{~L}\right) /(\partial \mathrm{z} \partial \mathrm{y})\right) \mathrm{Zdx} \\
+2 \mathrm{x}\left(\left(\partial^{2} \mathrm{~L}\right) /(\partial \mathrm{z} \partial \mathrm{z})\right) \mathrm{Zdx}-\left(\left(\partial^{2} \mathrm{~L}\right) /(\partial \mathrm{z} \partial \mathrm{x})\right) \mathrm{Zdy} .
\end{aligned}
$$

Energy function and its differential are like the following:

$$
\begin{aligned}
\mathrm{E}_{\mathrm{L}} & =\phi(\mathrm{L})-\mathrm{L} \\
& =\mathrm{X}((\partial \mathrm{L}) /(\partial \mathrm{y}))-\mathrm{X} 2 \mathrm{x}((\partial \mathrm{L}) /(\partial \mathrm{z}))+\mathrm{Y}((\partial \mathrm{L}) /(\partial \mathrm{x}))-\mathrm{L}
\end{aligned}
$$

and

$$
\begin{aligned}
\mathrm{dE}_{\mathrm{L}}= & ((\partial /(\partial \mathrm{x})) \mathrm{dx}+(\partial /(\partial \mathrm{y})) \mathrm{dy}+(\partial /(\partial \mathrm{z})) \mathrm{dz})(\mathrm{X}((\partial \mathrm{L}) /(\partial \mathrm{y}))-\mathrm{X} 2 \mathrm{x}((\partial \mathrm{L}) /(\partial \mathrm{z}))+\mathrm{Y}((\partial \mathrm{L}) /(\partial \mathrm{x}))-\mathrm{L}) \\
& =\left(\left(\partial^{2} \mathrm{~L}\right) /(\partial \mathrm{x} \partial \mathrm{y})\right) \mathrm{Xdx}-2 \mathrm{x}\left(\left(\partial^{2} \mathrm{~L}\right) /(\partial \mathrm{x} \partial \mathrm{z})\right) \mathrm{Xdx}+\left(\left(\partial^{2} \mathrm{~L}\right) /(\partial \mathrm{x} \partial \mathrm{x})\right) \mathrm{Ydx}-((\partial \mathrm{L}) /(\partial \mathrm{x})) \mathrm{dx} \\
& +\left(\left(\partial^{2} \mathrm{~L}\right) /(\partial \mathrm{y} \partial \mathrm{y})\right) \mathrm{Xdy}-2 \mathrm{x}\left(\left(\partial^{2} \mathrm{~L}\right) /(\partial \mathrm{y} \partial \mathrm{z})\right) \mathrm{Xdy}+\left(\left(\partial^{2} \mathrm{~L}\right) /(\partial \mathrm{y} \partial \mathrm{x})\right) \mathrm{Ydy}-((\partial \mathrm{L}) /(\partial \mathrm{y})) \mathrm{dy} \\
& +\left(\left(\partial^{2} \mathrm{~L}\right) /(\partial \mathrm{z} \partial \mathrm{y})\right) \mathrm{Xdz}-2 \mathrm{x}\left(\left(\partial^{2} \mathrm{~L}\right) /(\partial \mathrm{z} \partial \mathrm{z})\right) \mathrm{Xdz}+\left(\left(\partial^{2} \mathrm{~L}\right) /(\partial \mathrm{z} \partial \mathrm{x})\right) \mathrm{Ydz}-((\partial \mathrm{L}) /(\partial \mathrm{z})) \mathrm{dz}
\end{aligned}
$$

If we use $\mathrm{i}_{\xi} \Phi_{\mathrm{L}}=\Phi_{\mathrm{L}}(\xi)$ we obtain the equations given by

$$
\begin{aligned}
& -\left(\left(\partial^{2} \mathrm{~L}\right) /(\partial \mathrm{x} \partial \mathrm{y})\right) \mathrm{Xdx}+\left(\left(\partial^{2} \mathrm{~L}\right) /(\partial \mathrm{x} \partial \mathrm{y})\right) \mathrm{Xdx}+2 \mathrm{x}\left(\left(\partial^{2} \mathrm{~L}\right) /(\partial \mathrm{x} \partial \mathrm{z})\right) \mathrm{Xdx}-2 \mathrm{x}\left(\left(\partial^{2} \mathrm{~L}\right) /(\partial \mathrm{x} \partial \mathrm{z})\right) \mathrm{Xdx} \\
& -\left(\left(\partial^{2} \mathrm{~L}\right) /(\partial \mathrm{x} \partial \mathrm{x})\right) \mathrm{Xdy}+\left(\left(\partial^{2} \mathrm{~L}\right) /(\partial \mathrm{y} \partial \mathrm{y})\right) \mathrm{Xdy}-2 \mathrm{x}\left(\left(\partial^{2} \mathrm{~L}\right) /(\partial \mathrm{y} \partial \mathrm{z})\right) \mathrm{Xdy}+\left(\left(\partial^{2} \mathrm{~L}\right) /(\partial \mathrm{z} \partial \mathrm{y})\right) \mathrm{Xdz} \\
& -2 \mathrm{x}\left(\left(\partial^{2} \mathrm{~L}\right) /(\partial \mathrm{z} \partial \mathrm{z})\right) \mathrm{Xdz}+\left(\left(\partial^{2} \mathrm{~L}\right) /(\partial \mathrm{x} \partial \mathrm{x})\right) \mathrm{Ydx}-\left(\left(\partial^{2} \mathrm{~L}\right) /(\partial \mathrm{y} \partial \mathrm{y})\right) \mathrm{Ydx}+2 \mathrm{x}\left(\left(\partial^{2} \mathrm{~L}\right) /(\partial \mathrm{y} \partial \mathrm{z})\right) \mathrm{Ydx} \\
& -\left(\left(\partial^{2} \mathrm{~L}\right) /(\partial \mathrm{y} \partial \mathrm{x})\right) \mathrm{Ydy}+\left(\left(\partial^{2} \mathrm{~L}\right) /(\partial \mathrm{y} \partial \mathrm{x})\right) \mathrm{Ydy}+\left(\left(\partial^{2} \mathrm{~L}\right) /(\partial \mathrm{z} \partial \mathrm{x})\right) \mathrm{Ydz}-\left(\left(\partial^{2} \mathrm{~L}\right) /(\partial \mathrm{z} \partial \mathrm{y})\right) \mathrm{Zdx} \\
& +2 \mathrm{x}\left(\left(\partial^{2} \mathrm{~L}\right) /(\partial \mathrm{z} \partial \mathrm{z})\right) \mathrm{Zdx}-\left(\left(\partial^{2} \mathrm{~L}\right) /(\partial \mathrm{z} \partial \mathrm{x})\right) \mathrm{Zdy}
\end{aligned}
$$


$=$

$\left(\left(\partial^{2} \mathrm{~L}\right) /(\partial \mathrm{x} \partial \mathrm{y})\right) \mathrm{Xdx}-2 \mathrm{x}\left(\left(\partial^{2} \mathrm{~L}\right) /(\partial \mathrm{x} \partial \mathrm{z})\right) \mathrm{Xdx}+\left(\left(\partial^{2} \mathrm{~L}\right) /(\partial \mathrm{x} \partial \mathrm{x})\right) \mathrm{Ydx}-((\partial \mathrm{L}) /(\partial \mathrm{x})) \mathrm{dx}$

$+\left(\left(\partial^{2} \mathrm{~L}\right) /(\partial \mathrm{y} \partial \mathrm{y})\right) \mathrm{Xdy}-2 \mathrm{x}\left(\left(\partial^{2} \mathrm{~L}\right) /(\partial \mathrm{y} \partial \mathrm{z})\right) \mathrm{Xdy}+\left(\left(\partial^{2} \mathrm{~L}\right) /(\partial \mathrm{y} \partial \mathrm{x})\right) \mathrm{Ydy}-((\partial \mathrm{L}) /(\partial \mathrm{y})) \mathrm{dy}$

$+\left(\left(\partial^{2} \mathrm{~L}\right) /(\partial \mathrm{z} \partial \mathrm{y})\right) \mathrm{Xdz}-2 \mathrm{x}\left(\left(\partial^{2} \mathrm{~L}\right) /(\partial \mathrm{z} \partial \mathrm{z})\right) \mathrm{Xdz}+\left(\left(\partial^{2} \mathrm{~L}\right) /(\partial \mathrm{z} \partial \mathrm{x})\right) \mathrm{Ydz}-((\partial \mathrm{L}) /(\partial \mathrm{z})) \mathrm{dz}$.

From here

$$
\begin{aligned}
& -\left(\left(\partial^{2} \mathrm{~L}\right) /(\partial \mathrm{x} \partial \mathrm{y})\right) \mathrm{Xdx}+2 \mathrm{x}\left(\left(\partial^{2} \mathrm{~L}\right) /(\partial \mathrm{x} \partial \mathrm{z})\right) \mathrm{Xdx}-\left(\left(\partial^{2} \mathrm{~L}\right) /(\partial \mathrm{x} \partial \mathrm{x})\right) \mathrm{Xdy} \\
& -\left(\left(\partial^{2} \mathrm{~L}\right) /(\partial \mathrm{y} \partial \mathrm{y})\right) \mathrm{Ydx}+2 \mathrm{x}\left(\left(\partial^{2} \mathrm{~L}\right) /(\partial \mathrm{y} \partial \mathrm{z})\right) \mathrm{Ydx}-\left(\left(\partial^{2} \mathrm{~L}\right) /(\partial \mathrm{y} \partial \mathrm{x})\right) \mathrm{Ydy} \\
& -\left(\left(\partial^{2} \mathrm{~L}\right) /(\partial \mathrm{z} \partial \mathrm{y})\right) \mathrm{Zdx}+2 \mathrm{x}\left(\left(\partial^{2} \mathrm{~L}\right) /(\partial \mathrm{z} \partial \mathrm{z})\right) \mathrm{Zdx}-\left(\left(\partial^{2} \mathrm{~L}\right) /(\partial \mathrm{z} \partial \mathrm{x})\right) \mathrm{Zdy} \\
& +((\partial \mathrm{L}) /(\partial \mathrm{x})) \mathrm{dx}+((\partial \mathrm{L}) /(\partial \mathrm{y})) \mathrm{dy}+((\partial \mathrm{L}) /(\partial \mathrm{z})) \mathrm{dz} \\
& =0
\end{aligned}
$$

and

$$
\begin{aligned}
& \left(-\left(\left(\partial^{2} \mathrm{~L}\right) /(\partial \mathrm{x} \partial \mathrm{y})\right) \mathrm{X}+2 \mathrm{x}\left(\left(\partial^{2} \mathrm{~L}\right) /(\partial \mathrm{x} \partial \mathrm{z})\right) \mathrm{X}-\left(\left(\partial^{2} \mathrm{~L}\right) /(\partial \mathrm{y} \partial \mathrm{y})\right) \mathrm{Y}+2 \mathrm{x}\left(\left(\partial^{2} \mathrm{~L}\right) /(\partial \mathrm{y} \partial \mathrm{z})\right) \mathrm{Y}\right. \\
& \left.-\left(\left(\partial^{2} \mathrm{~L}\right) /(\partial \mathrm{z} \partial \mathrm{y})\right) \mathrm{Z}+2 \mathrm{x}\left(\left(\partial^{2} \mathrm{~L}\right) /(\partial \mathrm{z} \partial \mathrm{z})\right) \mathrm{Z}+((\partial \mathrm{L}) /(\partial \mathrm{x}))\right) \mathrm{dx}\left(\left(\partial^{2} \mathrm{~L}\right) /(\partial \mathrm{z} \partial \mathrm{z})\right) \mathrm{Z}+((\partial \mathrm{L}) /(\partial \mathrm{x})) \\
& +\left(-\left(\left(\partial^{2} \mathrm{~L}\right) /(\partial \mathrm{x} \partial \mathrm{x})\right) \mathrm{X}-\left(\left(\partial^{2} \mathrm{~L}\right) /(\partial \mathrm{y} \partial \mathrm{x})\right) \mathrm{Y}-\left(\left(\partial^{2} \mathrm{~L}\right) /(\partial \mathrm{z} \partial \mathrm{x})\right) \mathrm{Z}+((\partial \mathrm{L}) /(\partial \mathrm{y}))\right) \mathrm{dy}+((\partial \mathrm{L}) /(\partial \mathrm{z})) \mathrm{dz}=0, \\
& (\partial /(\partial \mathrm{t}))(-((\partial \mathrm{L}) /(\partial \mathrm{y}))+2 \mathrm{x}((\partial \mathrm{L}) /(\partial \mathrm{z}))) \mathrm{dx}+((\partial \mathrm{L}) /(\partial \mathrm{x})) \mathrm{dx}=0, \\
& (\partial /(\partial \mathrm{t}))(-((\partial \mathrm{L}) /(\partial \mathrm{x}))) \partial \mathrm{y}+((\partial \mathrm{L}) /(\partial \mathrm{y})) \mathrm{dy}=0, \\
& ((\partial \mathrm{L}) /(\partial \mathrm{z})) \mathrm{dz}=0,
\end{aligned}
$$

or

$$
\begin{aligned}
& {[(\mathrm{X}(\partial /(\partial \mathrm{x}))+\mathrm{Y}(\partial /(\partial \mathrm{y}))+\mathrm{Z}(\partial /(\partial \mathrm{z})))(-((\partial \mathrm{L}) /(\partial \mathrm{y}))+2 \mathrm{x}((\partial \mathrm{L}) /(\partial \mathrm{z})))+((\partial \mathrm{L}) /(\partial \mathrm{x}))] \mathrm{dx}} \\
& +[(\mathrm{X}(\partial /(\partial \mathrm{x}))+\mathrm{Y}(\partial /(\partial \mathrm{y}))+\mathrm{Z}(\partial /(\partial \mathrm{z})))(-((\partial \mathrm{L}) /(\partial \mathrm{x})))+((\partial \mathrm{L}) /(\partial \mathrm{y}))] \mathrm{dy} \\
& +((\partial \mathrm{L}) /(\partial \mathrm{z})) \mathrm{d} \mathrm{z}=0
\end{aligned}
$$

Considering the curve $\alpha=(\mathrm{x}, \mathrm{y}, \mathrm{z})$, an integral curve of $\xi$, i.e. $\xi(\alpha(\mathrm{t}))=\dot{\alpha}(t)=(\partial \alpha) /(\partial \mathrm{t})$.

Then, we can find the equations as follows:

$$
\begin{aligned}
& \xi(-((\partial \mathrm{L}) /(\partial \mathrm{y}))+2 \mathrm{x}((\partial \mathrm{L}) /(\partial \mathrm{z}))) \mathrm{dx}+((\partial \mathrm{L}) /(\partial \mathrm{x})) \mathrm{dx} \\
& +\xi(-((\partial \mathrm{L}) /(\partial \mathrm{x}))) \mathrm{dy}+((\partial \mathrm{L}) /(\partial \mathrm{y})) \mathrm{dy}+((\partial \mathrm{L}) /(\partial \mathrm{z})) \mathrm{dz}=0 \\
& (\partial /(\partial \mathrm{t}))(-((\partial \mathrm{L}) /(\partial \mathrm{y}))+2 \mathrm{x}((\partial \mathrm{L}) /(\partial \mathrm{z}))) \mathrm{dx}+((\partial \mathrm{L}) /(\partial \mathrm{x})) \mathrm{dx} \\
& +(\partial /(\partial \mathrm{t}))(-((\partial \mathrm{L}) /(\partial \mathrm{x}))) \mathrm{dy}+((\partial \mathrm{L}) /(\partial \mathrm{y})) \mathrm{dy}+((\partial \mathrm{L}) /(\partial \mathrm{z})) \mathrm{dz}=0 \\
& (\partial /(\partial \mathrm{t}))(-((\partial \mathrm{L}) /(\partial \mathrm{y}))+2 \mathrm{x}((\partial \mathrm{L}) /(\partial \mathrm{z}))) \mathrm{dx}+((\partial \mathrm{L}) /(\partial \mathrm{x})) \mathrm{dx}=0 \\
& (\partial /(\partial \mathrm{t}))(-((\partial \mathrm{L}) /(\partial \mathrm{x}))) \mathrm{dy}+((\partial \mathrm{L}) /(\partial \mathrm{y})) \mathrm{dy}=0 \\
& ((\partial \mathrm{L}) /(\partial \mathrm{z})) \mathrm{dz}=0
\end{aligned}
$$


or

$$
\begin{aligned}
& \text { dif1. }(\partial /(\partial \mathrm{t}))((\partial \mathrm{L}) /(\partial \mathrm{y}))+(\partial /(\partial \mathrm{t}))(2 \mathrm{x}((\partial \mathrm{L}) /(\partial \mathrm{z})))+(\partial \mathrm{L}) /(\partial \mathrm{x})=0, \\
& \text { dif2. }-(\partial /(\partial \mathrm{t}))(((\partial \mathrm{L}) /(\partial \mathrm{x})))+((\partial \mathrm{L}) /(\partial \mathrm{y}))=0, \\
& \text { dif3. }((\partial \mathrm{L}) /(\partial \mathrm{z})) \mathrm{dz}=0
\end{aligned}
$$

such that these equations are called Euler-Lagrange Equations constructed on 3-dimensional normal almost-paracontact metric manifolds and thus the triple $\left(\mathrm{M}, \Phi_{\mathrm{L}}, \xi\right)$ is named as a mechanical system on 3-dimensional normal almost-paracontact metric manifolds $(\mathrm{M}, \mathrm{g}, \phi)$.

\section{Hamiltonian Mechanical SySTEMS}

We, using Lemma 2, present Hamilton equations and Hamiltonian mechanical systems for quantum and classical mechanics constructed on 3-dimensional normal almost-paracontact metric manifolds.

Proposition 2. Let $\left(\mathrm{M}, \mathrm{g}, \phi^{*}\right)$ be the on 3-dimensional normal almost paracontact metric manifolds. Suppose that the structures, a Liouville form and a 1-form on 3-dimensional normal almostparacontact metric manifolds are shown by $\phi^{*}, \Omega$ and w, respectively. Consider a 1-form such that

$$
\mathrm{w}=(1 / 2)[\mathrm{dx}+\mathrm{dy}+\mathrm{dz}]
$$

Then the following differential equations are obtained.

$$
\begin{aligned}
& \text { dif4. }(\mathrm{dx}) /(\mathrm{dt})=(\partial \mathrm{H}) /(\partial \mathrm{z}), \\
& \text { dif5. }(\mathrm{dy}) /(\mathrm{dt})=0, \\
& \text { dif6. }(\mathrm{dz}) /(\mathrm{dt})=(\partial \mathrm{H}) /(\partial \mathrm{x}) .
\end{aligned}
$$

Proof: Then we obtain the Liouville form as follows:

$$
\begin{aligned}
\Omega=\phi^{*}(\mathrm{w}) & =(1 / 2)\left[\phi^{*}(\mathrm{dx})+\phi^{*}(\mathrm{dy})+\phi^{*}(\mathrm{dz})\right] . \\
& =(1 / 2)[\mathrm{dx}+\mathrm{dy}-2 \mathrm{xdz})]
\end{aligned}
$$

and

$$
\mathrm{d}(\Omega)=((\partial /(\partial \mathrm{x})) \mathrm{dx}+(\partial /(\partial \mathrm{y})) \mathrm{dy}+(\partial /(\partial \mathrm{z})) \mathrm{dz})(1 / 2)[\mathrm{dx}+\mathrm{dy}-2 \mathrm{xdz}] .
$$

Let's calculate $\Phi$ from here.

$$
\begin{aligned}
\Phi=-\mathrm{d}(\Omega) & =-(1 / 2)[((\partial(1)) /(\partial \mathrm{x})) \mathrm{d} x \wedge \mathrm{dx}+((\partial 1) /(\partial \mathrm{x})) \mathrm{dx} \wedge \mathrm{dy}-((\partial 2 \mathrm{x}) /(\partial \mathrm{x})) \mathrm{dx} \wedge \mathrm{dz} \\
& +((\partial(1)) /(\partial \mathrm{y})) \mathrm{dy} \wedge \mathrm{dx}+((\partial 1) /(\partial \mathrm{y})) \mathrm{dy} \wedge \mathrm{dy}-((\partial 2 \mathrm{x}) /(\partial \mathrm{y})) \mathrm{dy} \wedge \mathrm{dz} \\
& +((\partial(1)) /(\partial \mathrm{z})) \mathrm{dz} \wedge \mathrm{dx}+((\partial 1) /(\partial \mathrm{z})) \mathrm{dz} \wedge \mathrm{dy}-((\partial 2 \mathrm{x}) /(\partial \mathrm{z})) \mathrm{dz} \wedge \mathrm{dz}, \\
= & -1 / 2[0 \mathrm{~d} x \wedge \mathrm{dx}+0 \mathrm{dx} \wedge \mathrm{dy}-2 \mathrm{dx} \wedge \mathrm{dz} \\
& +0 \mathrm{dy} \wedge \mathrm{dx}+0 \mathrm{dy} \wedge \mathrm{dy}-0 \mathrm{dy} \wedge \mathrm{dz} \\
& +0 \mathrm{dz} \wedge \mathrm{dx}+0 \mathrm{dz} \wedge \mathrm{dy}-0 \mathrm{dz} \wedge \mathrm{dz}] \\
= & \mathrm{dx} \wedge \mathrm{dz} .
\end{aligned}
$$

It is well known that if $\Phi$ is a closed on 3-dimensional normal almost-paracontact metric manifolds $\left(\mathrm{M}, \mathrm{g}, \phi^{*}\right)$, then $\Phi$ is also a symplectic structure on $\left(\mathrm{M}, \mathrm{g}, \phi^{*}\right)$. Therefore the 2-form $\Phi$ indicates the 
canonical symplectic form and derived from the 1 -form $\Omega$ to find to mechanical equations. Then the 2-form is calculated as $\Phi=\mathrm{dx} \wedge \mathrm{dz}$.

Take a vector field $\mathrm{Z}_{\mathrm{H}}$ so that called to be Hamiltonian vector field associated with Hamiltonian energy $\mathrm{H}$ and determined by

$$
\mathrm{Z}_{\mathrm{H}}=\mathrm{X}(\partial /(\partial \mathrm{x}))+\mathrm{Y}(\partial /(\partial \mathrm{y}))+\mathrm{Z}(\partial /(\partial \mathrm{z}))
$$

So, we have

$$
\begin{aligned}
i_{Z_{H}} \varphi & =\varphi\left(Z_{\mathrm{H}}\right) \\
= & (\mathrm{dx} \wedge \mathrm{dz})(\mathrm{X}(\partial /(\partial \mathrm{x}))+\mathrm{Y}(\partial /(\partial \mathrm{y}))+\mathrm{Z}(\partial /(\partial \mathrm{z}))) \\
& =\mathrm{Xdz}-\mathrm{Zdx} .
\end{aligned}
$$

Furthermore, the differential of Hamiltonian energy $\mathrm{H}$ is obtained by

$$
\mathrm{dH}=\mathrm{X}((\partial \mathrm{H}) /(\partial \mathrm{x}))+\mathrm{Y}((\partial \mathrm{H}) /(\partial \mathrm{y}))+\mathrm{Z}((\partial \mathrm{H}) /(\partial \mathrm{z}))
$$

From $i_{L_{H}} \varphi=\mathrm{dH}$, the Hamiltonian vector field is found as follows:

$$
\mathrm{Z}_{\mathrm{H}}=((\partial \mathrm{H}) /(\partial \mathrm{z}))(\partial /(\partial \mathrm{x}))+((\partial \mathrm{H}) /(\partial \mathrm{x}))(\partial /(\partial \mathrm{z}))
$$

Consider the curve and its velocity vector $\alpha: I \subset \mathbb{R} \rightarrow M, \alpha(t)=(x(t), y(t), z(t))$,

$$
\dot{\alpha}(t)=((\mathrm{dx}) /(\mathrm{dt}))(\partial /(\partial \mathrm{x}))+((\mathrm{dy}) /(\mathrm{dt}))(\partial /(\partial \mathrm{y}))+((\mathrm{dz}) /(\mathrm{dt}))(\partial /(\partial \mathrm{z}))
$$

such that an integral curve of the Hamiltonian vector field $Z_{H}$, i.e., $Z_{H}(\alpha(t))=(\partial \alpha) /(\partial t), t \in I$, t shows the time.

$$
((\partial \mathrm{H}) /(\partial \mathrm{z}))(\partial /(\partial \mathrm{x}))+((\partial \mathrm{H}) /(\partial \mathrm{x}))(\partial /(\partial \mathrm{z}))=((\mathrm{dx}) /(\mathrm{dt}))(\partial /(\partial \mathrm{x}))+((\mathrm{dy}) /(\mathrm{dt}))(\partial /(\partial \mathrm{y}))+((\mathrm{dz}) /(\mathrm{dt}))(\partial /(\partial \mathrm{z}))
$$

Then, we can be found the following equations;

$$
\begin{aligned}
& \text { dif4. }(\mathrm{dx}) /(\mathrm{dt})=(\partial \mathrm{H}) /(\partial \mathrm{z}), \\
& \text { dif5. }(\mathrm{dy}) /(\mathrm{dt})=0, \\
& \text { dif6. }(\mathrm{dz}) /(\mathrm{dt})=(\partial \mathrm{H}) /(\partial \mathrm{x}) .
\end{aligned}
$$

Hence, the equations introduced in are named Hamilton equations on 3-dimensional normal almostparacontact metric manifolds and then the triple $\left(\mathrm{M}, \Phi, \mathrm{X}_{\mathrm{H}}\right)$ is said to be a Hamiltonian mechanical system on $\left(\mathrm{M}, \mathrm{g}, \phi^{*}\right)$.

\section{EquATIONS SOLVING WiTH COMPUTER}

The location of each object in space represented by three dimensions in physindcal space. These three dimensions can be labeled by a combination of three chosen from the terms length, width, height, depth, mass, density and breadth. These found (17) and (31) are partial differential equations on 3dimensional normal almost-paracontact metric manifolds such that it solved with Maple computation program.

Example 3. Let's solve the system of differential equations (17) by Maple. Euler-Lagrangian equations implicit solution for special values of $F_{1}(t)=t, F_{2}\left(y+x=y+x, F_{3}(y-x)=y-x\right.$, 
(1) $\mathrm{L}(\mathrm{x}, \mathrm{y}, \mathrm{z}, \mathrm{t})=\mathrm{F}_{1}(\mathrm{t})+\exp (\mathrm{t}) * \mathrm{~F}_{2}(\mathrm{y}+\mathrm{x})+\exp (-\mathrm{t}) * \mathrm{~F}_{3}(\mathrm{y}-\mathrm{x})$,

$$
=\mathrm{t}+\exp (\mathrm{t}) *(\mathrm{y}+\mathrm{x})+\exp (-\mathrm{t}) *(\mathrm{y}-\mathrm{x})
$$

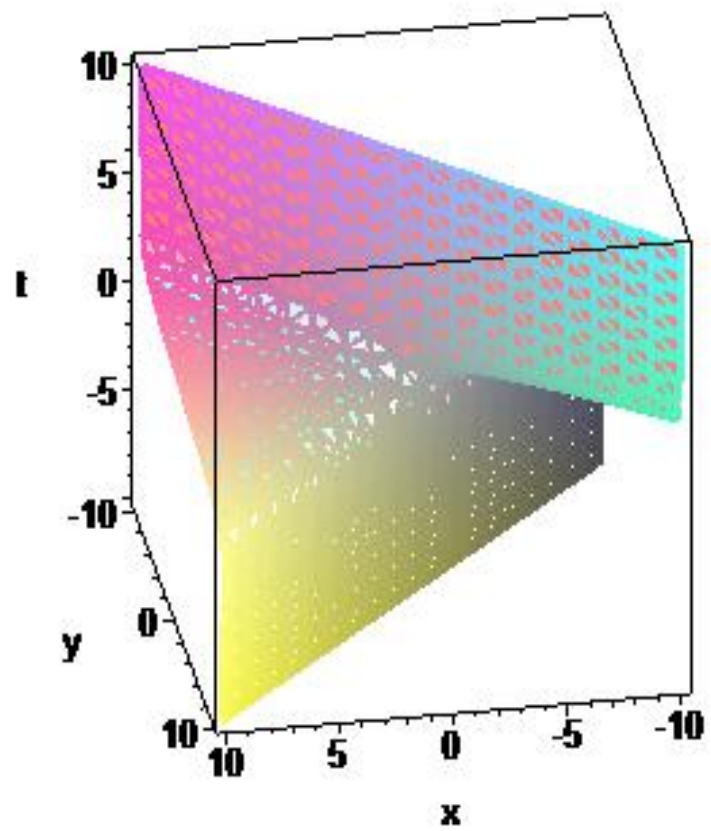

\section{Graph 1}

Example 4. Hamilton equations: implicit solution (31).

(2) $H(x, y, z, t)=z * \exp (t)+x-F_{1}(y, t)$

for special values of $x(t)=\exp (t), z(t)=t$ and $F_{1}(y, t)=0$

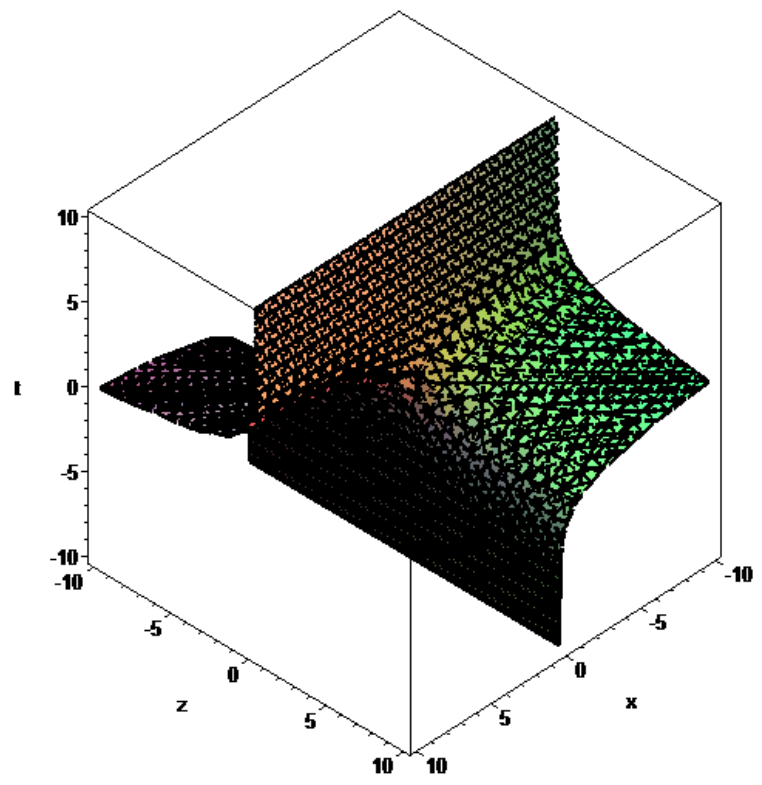

Graph 2 


\section{DISCUSSION}

It is well-known that a classical field theory explains the study of how one or more physical fields interact with matter which is used quantum and classical mechanics of physics branches. Also, the classical theory of electromagnetism deals with electric and magnetic fields and their interaction with each other and with charges and currents. An electromagnetic field is a physical field produced by electrically charged objects. How the movement of objects in electrical, magnetically and gravitational fields force is very important. For instance, on a weather map, the surface wind velocity is defined by assigning a vector to each point on a map. So, each vector represents the speed and direction of the movement of air at that point.

In this study, the Euler-Lagrange and Hamilton mechanical equations (17) and (31) derived on 3dimensional normal almost-paracontact metric manifolds may be suggested to deal with problems in electrical, magnetical and gravitational fields for the path of movement (42) and (43) of defined space moving objects $[18,19]$.

\section{REFERENCES}

[1] Welyczko J., On Legendre curves in 3-dimensional normal almost contact metric manifolds, Soochow Journal of Mathematics, 33(4), (2007), 929-937.

[2] Tripathi M.M., Kılıc E., Perktas S.Y. and Keles S., Indefinite almost paracontact metric manifolds, International Journal of Mathematics and Mathematical Sciences, Volume 2010, 1-19.

[3] Atceken M., Warped product semi-invariant submanifolds in almost paracontact metric manifolds, Mathematica Moravica, 14(1), 2010, 15-21.

[4] Călin C., Crasmareanu M. and Munteanu M., Slant curves in 3-dimensional f-Kenmotsu manifolds, J. Math. Anal. Appl., 394, 2012, 1-9.

[5] C. Călin and M. Crasmareanu, Slant curves in 3-dimensional normal almost contact geometry, Mediterranean Journal of Mathematics, 10(2), 2013, 1067-1077.

[6] Kasap Z. and Tekkoyun M., Mechanical systems on almost para/pseudo-Kähler-Weyl manifolds, IJGMMP, 10(5), 2013, 1-8.

[7] Guvenc S. and Ozgur C., On slant curves in trans-Sasakian manifolds, Revista De La Uni on Matem Atica Argentina, 55(2), 2014, 81-100.

[8] Welyczko J., Slant curves in 3-dimensional normal almost paracontact metric manifolds, Mediterranean Journal of Mathematics, 11(3), 2014, 965-978.

[9] Calvaruso G. and Perrone D., Geometry of H-paracontact metric manifolds, arXiv:1307.7662v1, 2013, 116.

[10] McDu D. and Salamon D., J-Holomorphic Curves and Quantum Cohomology, https://barnard.edu/sites/ default/files/inline/jholsm_0.pdf, 1995.

[11] Nowaczyk N., Niediek J. and Firsching M., Basics of Complex Manifolds, 2009.

[12] Newlander A. and Nirenberg L., Complex analytic coordinates in almost complex manifolds, Ann. of Math., 65, (1957), 391-404.

[13] Griffiths D.J., Introduction to Electrodynamics. Prentice Hall. ISBN 0-13-805326-X, 1999.

[14] De Leon M. and Rodrigues P.R., Methods of Differential Geometry in Analytical Mechanics, North-Hol. Math. St., 152, Elsevier, 1989.

[15] Abraham R., Marsden J.E. and Ratiu T., Manifolds Tensor Analysis and Applications, Springer, 2001.

[16] Klein J., Escapes Variationnels et Mécanique, Ann. Inst. Fourier, Grenoble, 12, 1962.

[17] Ibord, A. The Geometry of Dynamics, Extracta Mathematicable, 11(1), 1996, 80-105.

[18] Thidé B., Electromagnetic Field Theory, Department of Astronomy and Space Physics Uppsala University, Sweden, 2012.

[19] Martín R.G., Electromagnetic Field Theory for Physicists and Engineers: Fundamentals and Applications, Asignatura: Electrodinámica, Físicas, Granada, (2007). 


\section{AUTHORS' BIOGRAPHY}

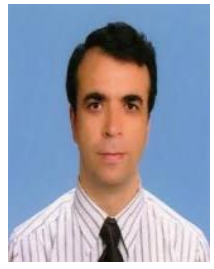

Dr. Zeki KASAP, He is working as a Academics Faculty of Education, Department of Elementary Mathematics Education in Pamukkale University, Denizli/TURKEY. His research arears of interest are Differential Geometry, Mathematical Physics, Analytical Mechanics, Dynamical Systems, Equations of Motion of Objects in Space and Mathematics Education. His papers more than 27 were published in various reputed National and International Journals. http://orcid.org/0000-0001-8912-7734, ResearcherID:K-3823-2015, https://pamukkale.academia.edu/zekikasap, Scopus Author ID: 43661468400, MathSciNet Author ID: 911058.

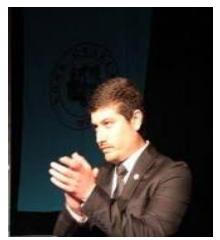

Oguzhan Çelik

The author is a free researcher.

He is a graduate.

Department of Mathematics, Institute of Science,

University of Çanakkale Onsekizmart,

Çanakkale/ TURKEY.

Citation: O. Çelik, Z. Kasap, "Mechanical Equations on Slant Curves in 3-dimensional Normal Almost Paracontact Metric Manifolds ", International Journal of Scientific and Innovative Mathematical Research, vol. 5, no. 9, p. 22-33, 2017., http://dx.doi.org/10.20431/2347-3142.0509002

Copyright: () 2017 Authors. This is an open-access article distributed under the terms of the Creative Commons Attribution License, which permits unrestricted use, distribution, and reproduction in any medium, provided the original author and source are credited. 